\title{
Detection methods of avian influenza - current and novel approaches
}

\author{
May Ye Yee ${ }^{1}$, Shaharum Shamsuddin², Quaza Nizamuddin Hassan Nizam ${ }^{4}$, Muhammad Redzwan Sidik ${ }^{3}$, Faizul \\ Fikri Mohd Yusop ${ }^{3}$, Faizah Hanim Mohd. Saeid ${ }^{3}$ and Ismail Aziah ${ }^{1 *}$
}

${ }^{1}$ Institute for Research in Molecular Medicine, University Sains Malaysia, 16150 Kubang Kerian, Kelantan, Malaysia ${ }^{2}$ School of Health Sciences, Health Campus, Universiti Sains Malaysia, 16150 Kubang Kerian, Kelantan, Malaysia.

${ }^{3}$ Veterinary Research Institute, 31400 Ipoh, Perak. Email: aziahismail@usm.my

Received 25 February 2019; Received in revised form 6 May 2019; Accepted 23 June 2019

\begin{abstract}
Avian influenza (AI), caused by the avian strain of influenza A virus (AIV) is one of the significant health concerns globally. Human infections with Al viruses were reported sporadically and often exhibited high mortality and morbidity rate. Al outbreaks also influenced the safety of the food supply and caused significant economic losses. Immediate control measures are required during Al outbreaks in poultry to prevent further viruses spreading. Hence, accurate, sensitive, and rapid detection methods are pivotal for decision making. Traditional methods of detection, such as virus isolation in embryonated chicken eggs, immuno-based methods, and nucleic acid amplification method, pose different limitations. These always grab the attention of researchers to improve existing methods or invent novel diagnostic approaches to compensate for the shortcoming of current methods applied. However, the method of choice is highly dependent on the availability of facilities and resources. Among the detection methods, reverse transcription-polymerase chain reaction (RT-PCR) is the most favourable method used for detecting AIV. However, a constant review of the virus genome is crucial to maintain the assay's sensitivity. More comprehensive research and evaluation study are needed for new diagnostic approaches.
\end{abstract}

Keywords: Avian influenza, detection methods

\section{INTRODUCTION}

Avian influenza $(\mathrm{Al})$ is a serious problem, especially in the poultry industry, caused by infection of AIV. AIV is influenza $A$ virus (IFAV) adapted in avian species. IFAV is the only species under Alpha influenza virus genus of Orthomyxoviridae family (Monne et al., 2008). The viral genome consists of eight segmented, single-stranded RNA in negative polarity, which encodes different viral proteins such as RNA polymerase (PB1, PB2, and PA), nucleoprotein (NP), hemagglutinin (HA), neuraminidase (NA), matrix (M) protein and non-structural (NS) protein (Krammer et al., 2018). The structure of a virus is presented in Figure 1. The lipid envelope of virus studded with $\mathrm{HA}$ and NA protein and transversed by the M2 proteins. M1 proteins lie beneath the envelope and enclose the virion core, which consists of 8 segmented viral genomes. Each ribonucleic acid (RNA) segment is coated with RNA polymerase (PB1, PB2, and PA) and NP. HA is used for virus attachment and entry to the host cell. NA cleaves sialic acid from host cell receptors to enable the release of new viral progenies. Hence, these two proteins play a significant role in virus replication and are used for subtyping further the virus based on a different combination of $\mathrm{HA}$ and NA. To date, there are 18 $\mathrm{HA}$, and $11 \mathrm{NA}$ subtypes in all possible combinations have been discovered in all IFAVs. Wild aquatic birds are considered as a natural reservoir of the virus (Monne et al., 2008; Poovorawan et al., 2013; Vemula et al., 2016; Dziabowska et al., 2018).

Influenza A virus may sporadically infect humans that have direct or indirect contact with infected poultry or contaminated surfaces. It is further classified into two distinct pathotypes, low pathogenic AI (LPAI) and high pathogenic Al (HPAl), based on genetic characteristics and degree of disease severity and mortality (Chaharaein et al., 2009; Tahir et al., 2016). Infection by LPAI viruses may be asymptomatic or exhibit mild symptoms such as drops in egg production or ruffled feathers but HPAl viruses usually cause severe disease with high mortality and spread rapidly in poultry flocks due to the presence of multi-basic cleavage sites in $\mathrm{HA}$, rendering its high virulence by affecting multiple organs (Nur Adibah et al., 2017). Hence, HA is the key to virulence determination. Among $18 \mathrm{HA}$ subtypes, only AIV subtype $\mathrm{H} 5$ and $\mathrm{H} 7$ are considered as HPAl virus (Lee et al., 2001; Monne et al., 2008; Tahir et al., 2016). But, not all H5 and H7 subtype of AIV is high pathogenic. However, LPAI viruses may 
transform into a highly pathogenic form due to the accumulation of genetic mutation in the HA gene segment, particularly in the HA cleavage sites (HACS).

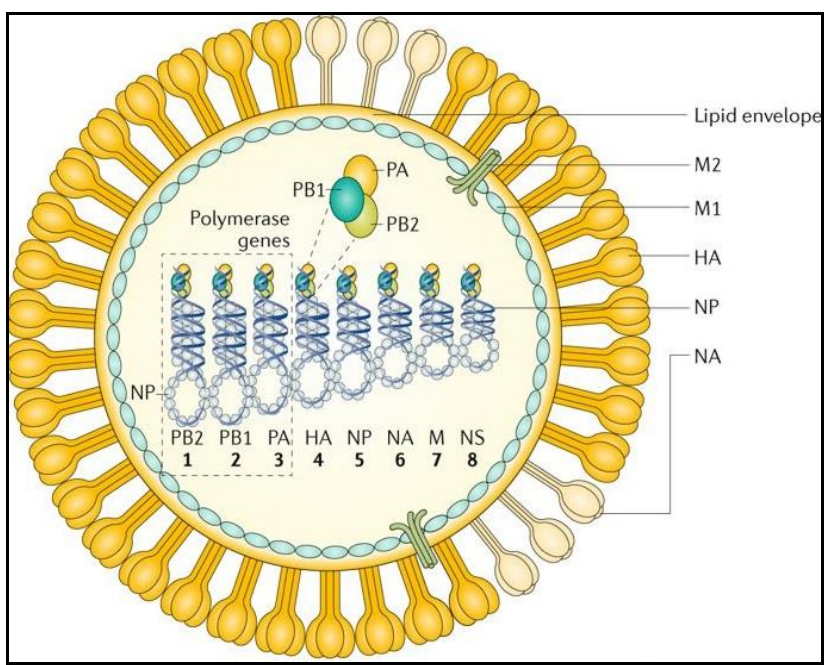

Figure 1: Structure of AIV. [Source: (Krammer et al., 2018)].

Influenza A virus subtype $\mathrm{H} 5 \mathrm{~N} 1$ (H5N1 virus) has been circulating in the poultry as LPAl virus before 1997 and mutated into a highly pathogenic form which led to devastating consequences in domestic fowl and caused 18 human infections with six fatal cases in Hong Kong during 1997 (WHO, 2005). Since then, this HPAl virus spread rapidly to the many Asian countries such as South Korea, China, Japan, Vietnam, Thailand, Laos, Indonesia, and Malaysia, causing tremendous economic losses in the poultry industry (WHO, 2005; Nur Adibah et al., 2017). Human infections with AIV subtype H7N9 (H7N9 virus) were reported in China during the early of 2013 (CDC, 2018; Jiao et al., 2018). Infected patients were severely ill with high fatality risk (Yu et al., 2013) but this virus may shed in poultry without any symptoms or with less severe illnesses (WHO, 2017; Jiao et al., 2018). Since then, many human infected cases were reported frequently in China despite no evidence of sustained human-to-human transmission. $\mathrm{H} 7 \mathrm{~N} 9$ virus also poses similar health threat like HPAI H5N1 virus as the cumulative cases of human infection with this virus since 2013 till 2017 has hit to a number similar to the cases caused by $\mathrm{H} 5 \mathrm{~N} 1$ virus (Jiao et al., 2018). It was also reported that LPAI H7N9 virus has undergone mutation and transformed into HPAl virus (OIE, 2018). In addition, AIV subtype H9N2 (H9N2 virus) is another subtype that commonly isolated from poultry and mammals and was reported to cause human infections sporadically (Butt et al., 2005). Therefore, subtype $\mathrm{H} 5, \mathrm{H} 7$, and $\mathrm{H} 9$ are usually prioritized in AIV subtyping. AIV is impossible to be eradicated. This virus is circulating in wild birds, and poultry regularly causes outbreaks and influences poultry production as all products including uninfected poultry in the vicinity of infectious locations to need to be destroyed to ensure the safety of consumption and usage. Detection of AIV is the first line of defence against AIV so that appropriate measures can be taken immediately to minimize further virus spreading. Several detection methods are available and adapted to be used in various reference laboratories to provide diagnostic results rapidly, especially during outbreak season. Scientists have continually improved existing diagnostic methods to overcome issues of sensitivity and simplify complicated procedures. Besides, new diagnostic platforms were also invented along with existing technologies despite less likely adapted in diagnostic laboratories. This article aims to provide a brief overview of existing and underdevelopment AIV detection methods with summarized principle and their application in $\mathrm{Al}$ detection and surveillance system.

\section{CURRENT METHODS OF DETECTION}

Various methods have been recommended by the World Health Organization (WHO) and the World Organisation of Animal Health (OIE) for AIV detection. These methods are either aim to detect the viruses directly or antibodies against the virus. The detection methods include virus isolation, hemagglutination assay, hemagglutination inhibition assay, virus neutralization test, enzyme-linked immunosorbent assay (ELISA), agar gel immunodiffusion (AGID), immunofluorescence tests, conventional or realtime reverse-transcriptase polymerase chain reaction (qRT-PCR) and rapid influenza detection test (RIDT).

\section{Virus isolation}

Isolation of AIV from samples is the gold standard and most traditional method of AIV detection since it is introduced in the 1940s (Vemula et al., 2016; Dziabowska et al., 2018). Various types of samples such as respiratory tracts and cloacal swabs, fresh faeces, pool of internal organs as well as water from the contaminated environments can be inoculated into 9 to 11-day-old specific-pathogenic-free embryonated chicken egg via allantoic route and incubated for about 4 days at $37{ }^{\circ} \mathrm{C}$ (Krauss et al., 2012). Live viruses in the sample replicate in cells of the chorioallantoic membrane, release by budding into the allantoic cavity containing allantoic fluid. The allantoic fluid is collected once inoculated egg is found dead. The abundance of sialic acid receptor in $\alpha-$ 2,3 configuration in cells lining the allantoic cavity favours AIV replication (Eisfeld et al., 2014). HPAI viruses can kill the embryo within $24 \mathrm{~h}$ (Woolcock, 2008). Another method is by using mammalian cell lines such as MadinDarby Canine Kidney (MDCK) cells and Vero cells derived from African Green Monkey Kidney, in which viruses are detected by cytopathic effects (CPE) (Krauss et al., 2012). However, it has been reported that replicating AIV with mammalian cell lines was less efficient compared to embryonated chicken eggs due to the presence of $\alpha-2,6$ linked sialic acid receptors on the cells (Eisfeld et al., 2014). Human or swine influenza viruses are more likely to be propagated using mammalian cell lines. This method is highly sensitive for 
detecting live AIV from samples. It is required to generate high virus titre for downstream analysis. However, continuous supply of embryonated eggs or maintenance of cells is needed for virus propagation. Biosafety level (BSL) II or III laboratory facility is also required while performing virus inoculation, rendering it impractical in the resource-limited laboratory setting. Besides this, TAT is too long as the virus isolates will be further analyzed with other techniques for virus confirmation (Woolcock, 2008; Shi et al., 2015). Also, false negative results may obtain in case of non-infectious viruses shed in the sample because only infectious viruses will replicate. This method is unsuitable for rapid diagnosis, but it has still been used in parallel with PCR assay in some laboratories. Furthermore, it also produces viruses source required for vaccines production and genetic characterization for surveillance purpose.

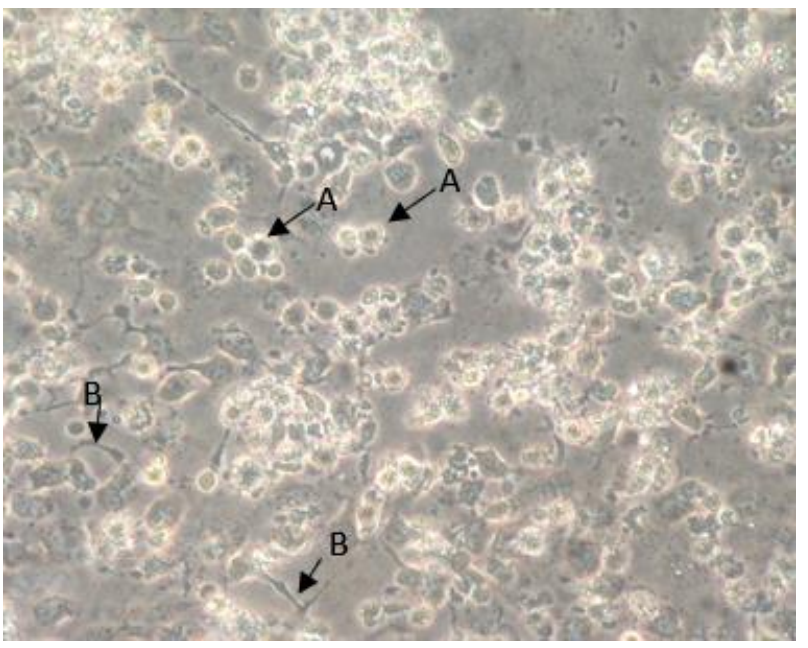

Figure 2: CPE of MDCK cells induced by AIV obtained under $10 \times$ magnification. AIV caused rounding of infected cells and lead to the formation of cytoplasmic bridges across intercellular spaces, resulting loss of normal cell architecture. A: cell rounding. B: Cytoplasmic bridges. (Infection of MDCK cells with H5N1 viruses)

\section{Hemagglutination assay}

Positive virus isolation is usually followed by hemagglutination assay, which aims to detect and quantitate the hemagglutinating virus in hemagglutination unit (HAU). HA present on the surface of a virus can bind and agglutinate red blood cells (RBCs). The agglutinated RBCs will form lattices and remain distributed in the suspension, this giving a smeared reddish appearance. In contrast, RBCs will settle down at the bottom of a well, forming a sharp dot of cells (Eisfeld et al., 2014). This assay detects the presence of viral HA protein, hence, capable of detecting live, degraded, and non-infectious forms of viruses. However, hemagglutination assay is not specific to AIV because other viruses such as Newcastle disease viruses also possess hemagglutinating activity.
Therefore, other aetiological agents should be considered in a positive result, and subsequent identification tests need to be carried out to confirm AIV (Killian, 2008). The origin of RBCs used to perform this assay is also important as the hemagglutinating activity is influenced by the species-specific expression of $\alpha-2,3$ and $\alpha-2,6$ linked sialic acid receptors on RBCs (Eisfeld et al., 2014). Hence, RBCs of avian origin should be used for determining HAU of AIV.

\section{Hemagglutination inhibition (HI) assay}

Hemagglutination inhibition $(\mathrm{HI})$ assay is one of the serological methods generally used for subtyping AIV in virus isolates by detecting HA-specific antibodies raised against HA of a particular subtype. Serum sample contains specific anti-HA antibodies will attach to the test viruses, preventing viruses from agglutinating RBCs. Complete settling of RBCs will result in a distinct button at the bottom of a well. The result is reported as $\mathrm{HI}$ titre obtained from reciprocal of the highest serum dilution that produces complete $\mathrm{HI}$ (Shi et al., 2015; Vemula et al., 2016). This method not only can subtype unknown AIV but also can evaluate the antigenic relationship between different clades of the same AIV subtype (Pedersen, 2008; Thi Nguyen et al., 2018). It is also essential for vaccine strain selection, production, and efficacy assessment (Wilson et al., 2017; Thi Nguyen et al., 2018). $\mathrm{HI}$ assay is inexpensive and easy to perform. It has high accuracy for virus subtyping. However, its sensitivity is controversial (Vemula et al., 2016). The HI titer is highly dependent on the antigenic relatedness between antibody in the serum and antigen source according (Pedersen, 2008). The amino acid difference exists between AIV of the same subtype, and this could affect the avidity of antibodies to the antigen source, which in turn affect the assay sensitivity. Especially for the H5N1 virus, the antibody against the virus from different clades may unreactive to the antigen source of $\mathrm{H} 5$ subtype. Besides, novel HA subtype of AIV could not be identified as this assay is only available for existing HA subtypes. Further testing is still required to determine the hemagglutinating agent.

\section{Viral neutralization (VN) assay}

Viral neutralization (VN) assay is an alternative method of $\mathrm{HI}$ assay which aims to detect specific anti-HA antibodies in serum samples using live, infectious viruses. Antibodies present in the sample will react with the test viruses, neutralizing viral infection. Residual viral infectivity is determined by adding a reaction mixture to MDCK cells. The absence of CPE indicates complete neutralization of viruses. Alternatively, ELISA is performed to detect NP of AIV in virus-infected cells (WHO, 2010). Plaque reduction assay is also another option to measure viral infectivity, but it is not commonly used due to the difficulty of result interpretation, which may lead to an inaccurate and imprecise result. Imaging device was used to quantify total infected cells population 
instead of counting plaques formed with naked eyes (Lin et al., 2016). This assay is very sensitive in case of low antibody titer and specific in detecting functional HAspecific antibodies compared to $\mathrm{HI}$ assay (Klimov et al., 2012; Shi et al., 2015; Tavakoli et al., 2017; Dziabowska et al., 2018). Unlike $\mathrm{HI}$ assay, it is readily available for the new strain of virus upon recognition as the infections can be used temporarily for detecting new cases (Klimov et al., 2012). However, it is laborious because it requires continuous maintenance of MDCK cells culture and infectious viruses. Besides, a BSL III laboratory facility and trained personnel are also needed for safe manipulation of HPAl viruses. Hence, it is less favourable for routine diagnostic application.

\section{Neuraminidase-inhibition (NAI) assay}

Neuraminidase (NA) is an antigenic determinant secondary to HA. The method of quantitating sialic acid was first described by Aminoff (1961) and modified by Van Deusen et al. (1983) into microtiter plate platform to determine NA subtype of IFAV (Van Deusen et al., 1983). $\mathrm{NAI}$ assay was used in conjunction with $\mathrm{HI}$ assay for subtyping of AIV. This assay detects either specific NA antigen or anti-NA antibodies using reference antisera or antigen. NA activity is detected by adding fetuin substrate, a glycosylated protein in which NA will act on, releasing free sialic acids or $\mathrm{N}$-acetyl neuraminic acid (NANA). Free NANA is then oxidized by periodate, forming $\beta$ formylpyruvic acid which reacts with thiobarbituric acid in subsequent hot acidic reaction, forming pink chromophores. Results are read from the colour developed in comparison with the positive control (Pedersen, 2008; Sandbulte et al., 2009; Terregino and Capua, 2009). NAl assay for the use of NA subtype identification is replaced by molecular method nowadays. Lengthy test procedure and utilization of hazardous chemicals render this assay impractical for routine diagnosis (Sandbulte et al., 2009). Modified NAl assay using either fluorescence [2'-(4-Methylumbelliferyl)- $\alpha-D-N$ acetylneuraminic acid (MUNANA)] or chemiluminescence [1,2-dioxetane (NA-STAR)] substrate is adapted for determining the effectiveness of NA inhibitors (WHO, 2011; Leang and Hurt, 2017). Commercial kits of NAI assay are available for determining antiviral susceptibility of IFAV. NAI assay is required to detect viral resistance to NA inhibitors due to amino acid substitutions in NA to ensure the treatment efficacy.

\section{ELISA}

ELISA is a well-known technique in serology introduced in 1990 , available in a microtiter plate or strip format, to detect target antibodies or antigens (Vemula et al., 2016). ELISA depends on the formation of antigen-antibody complexes, which are recognized by enzyme-linked antibodies that cleaves substrates into measurable products in a direct, indirect, or sandwich format. It is usually used for general detection of AIV. Antibodies against NP are often the target of ELISA due to the highly conservative properties in all IFAV species (OIE, 2015). Indirect ELISA is highly dependent on the species specificity between the target antibodies and enzymeconjugated secondary antibodies; thus, both blocking ELISA (bELISA) and competitive ELISA (cELISA) are preferred to detect anti-AIV antibodies regardless of species constraint (Vandalen et al., 2009). Both bELISA and CELISA work on the same principle that detects target antibodies by competition with enzyme-linked antibodies which bind to the same target antigen. The presence of target antibodies is represented by the absence or reduced light absorbance by spectrophotometer. They are slightly different in the procedure where positive test serum and competitive enzyme-conjugated antiserum react with antigen binding sites sequentially in bELISA but simultaneously in cELISA. Sensitivity and specificity of bELISA and cELISA are reported to be higher compared with AGID and comparable with $\mathrm{HI}$ assay (Vandalen et al., 2009). Currently, many commercial ELISA kits are targeting either anti-NP antibodies or NP in indirect, competitive, and sandwich format are available. It is suitable for high throughput-screening of AIV infection and evaluation of vaccine-induced immune response in poultry farms. Test results can be obtained within 15 minutes. Furthermore, ELISA kits for subtyping such as ID Screen Influenza subtype-specific kits are also available. Nevertheless, the molecular method is still preferable compared with ELISA due to sensitivity issue (Vemula et al., 2016; Dziabowska et al., 2018).

\section{AGID test}

AGID test is generally used for screening anti-AIV antibodies in poultry. Antibodies against NP and M protein are the target of this test. Hence, this is a general detection method for AIV infection by not providing any subtype information. This test utilizes a gel matrix to detect antigen-antibody complexes in the form of a precipitation line. Reference antigens are dispensed into a well cut in the centre of an agar gel, surrounded by wells filled with positive antiserum, test serum, and negative antiserum. Specific antibodies present in the serum will react with the antigens during overnight incubation, forming visible precipitin line at the interface. Visibility of the line formed is highly dependent on the concentration of the antibody present. Low antibody titre may lead to a false negative result. Besides, it has limited use in AIV detection, especially in wild birds due to the variability of antibodies produced between avian species. This test also relies on the production of precipitating antibodies, which is absent in some birds (Vandalen et al., 2009; OIE, 2015). Furthermore, the sensitivity of this test was demonstrated to be lower than the commercial bELISA (Brown et al., 2009). However, the AGID test is still practiced for serological screening of AIV infection in domesticated chicken and turkeys flocks (OIE, 2015) as it is a low-cost test. It is usually combined with $\mathrm{HI}$ assay for AIV identification for non-urgent cases. 


\section{Immunofluorescence test (IFT)}

Immunofluorescence test (IFT) detects viral antigens present in infected cells/tissues using fluorescencetagged antibodies (FA). This technique was described by Coons et al. (1941) and first applied for the detection of human influenza virus infection in ferret model in 1955 (Liu, 1955). This test can either perform directly with specific anti-AIV antibodies linked to a fluorescent dye or an indirect manner with secondary FA. Fluorescencetagged viral antigens are then visualized under a fluorescence microscope. Direct IFT is easier to perform and able to generate results faster but less sensitive compared to indirect IFT (Van Zyl, 2006). IFT is suitable to detect viral antigens in respiratory specimens, tissues, or cell culture. It had been used to identify organ/tissue tropism of LPAI viruses (Mehdi Hadipour, 2009). Hence, it also plays an essential part in pathogenesis study of AIV. Commercial immunofluorescence reagent kits are available for respiratory identification viruses from clinical samples, but not solely for AIV detection. In comparison with immunohistochemical staining, IFT is readily simpler to perform; the results also generated more easily to be identified due to less background interference. However, the sensitivity of IFT is lower than RT-PCR. Skilled expertise is also needed to perform fluorescence microscopy for results confirmation.

\section{PCR}

PCR was developed by Kary Mullis in 1983 to generate many copies of genetic materials. Detection of AIV using PCR was done since 1991 (Shi et al., 2015). RT-PCR is commonly used to diagnose AIV infection and subtyping. It starts with first strand complementary DNA (cDNA) synthesis by reverse transcriptase. When RNase removes template RNA strand of the RNA-DNA hybrid, PCR starts and produces many copies of the target gene. $M$ gene is usually the target of virus detection while subtyping is achieved by targeting $\mathrm{HA}$ and NA gene. Some groups had utilized degenerate primers to detect HA and NA subtypes (Alvarez et al., 2008; OIE, 2015). $\mathrm{HA}$ is the main target of immune protection in the infected host system, the nature of the virus which replicates by employing RNA polymerase that lack of proofreading ability, causing amino acid differences to exist between HA of the same subtype and wobbles present in codon usage (Lee et al., 2001). Hence, primers design is challenging the as large amount of sequences available in gene bank need to be examined to design the best-suited primer. Degenerate primers could help in overcoming this issue and increase diagnostic sensitivity. RT-PCR can be either performed in one-step or two-step, where RT and amplification step occur in the same tube for one-step but separately in a two-step. One-step RT-PCR offers a quick set-up procedure and reduces sample handling. In twostep RT-PCR, amplification set-up is required after cDNA synthesis from viral RNA, possessing a risk of samples cross-contamination. Despite the flexibility and sensitivity offered by two-step RT-PCR, the one-step procedure is still a preferred choice for screening large quantities of samples, especially during outbreaks (Suarez et al., 2007). Multiplex one-step RT-PCR further speeds up the identification of three subtypes $(\mathrm{H} 5, \mathrm{H} 7$, and $\mathrm{H} 9)$ that cause significant effect in poultry as well as human health (Chaharaein et al., 2009).

Rapid evolution in technology leads to the invention of qRT-PCR. The principle of qRT-PCR is almost similar to traditional RT-PCR, but the method of detecting amplified DNA fragments is different. Accumulation of qRT-PCR products is detected 'real-time' utilizing fluorescence generated from cleaving of a fluorescently labelled probe (hydrolysis or TaqMan probe) or binding of intercalating dye (SYBR green) to amplified products. Hydrolysis probe is the most commonly used probe in probe-based qRTPCR (OIE, 2015). It is an oligonucleotide labelled with a fluorogenic reporter dye at the 5' end, and a quencher dye at the 3' end. Taq polymerase that exhibits 5' exonuclease activity will cleave the reporter dye from the probe during extension step. Fluorescence of the reporter dye at a specific wavelength will be detected by real-time PCR instrument.

Commercial qRT-PCR kits for AIV detection and subtyping are available in European and American countries. But, their suitability for use in Malaysia is yet to be evaluated because different viral lineages circulate in North America and Eurasia. qRT-PCR is a method widely used for AIV screening due to its high sensitivity and specificity (Spackman and Suarez, 2008; OIE, 2015). It also plays a vital role in AIV detection, especially during outbreak season because it enables high-throughput screening and exhibits shorter TAT. However, false negative results may obtain due to the presence of PCR inhibitors in samples or poor RNA quality (Shi et al., 2015). Incorporation of internal positive control into the reaction is highly recommended to ensure proper test run. Furthermore, PCR is prone to contamination from carryover PCR products, which leads to false positive results. Spacious laboratory setting is required for nucleic acid extraction, PCR set-up, and amplification to avoid misleading results. Primers also need to be continuously reviewed due to the high mutation rate of the viral genome.

\section{Sanger sequencing}

IFAVs constantly evolve through antigenic drift and shift. Genetic characterization of these viruses is one of the preventive measures of the potential outbreak by monitoring changes in the viral genome. Genome sequencing is a process to identify the nucleotide sequence of the gene of interest. It was traditionally done by Sanger sequencing which developed by Fred Sanger and his colleagues in 1977. Over time, modification of existing methods such as fluorescent labelling, capillary electrophoresis, and general automation make sequencing procedure less labour intensive together with a more straightforward data output. Sanger sequencing technique is also known as the chain-termination method, using components similar to PCR and an additional 
component, dideoxynucleotides (ddNTPs). Each type of ddNTPs is labelled with a different fluorophore, which terminates the elongation process once incorporated into a polymerizing strand, producing various sizes of DNA fragments with 3' end labelled with a fluorophore. These DNA fragments are subjected to capillary gel electrophoresis, which passes through a laser beam and illuminated in ascending order of size. A detector will detect the different wavelength of light emitted sequentially, conveying into a series of peaks of fluorescence intensity in a chromatogram where DNA sequence could be deduced. Sanger sequencing capable of determining DNA sequences up to $1000 \mathrm{bp}$ (Vemula et al., 2016). It is usually used after a positive RT-PCR to confirm the identity of amplicon obtained from an isolate. Deng et al. (2015) obtained full genome sequence of six internal genes (HA and NA were excluded) of IFAVs (including AIV subtype H7N9 dan H10N7) by producing M13 tagged-amplicon of each gene and sequenced the amplicons using M13 universal primer pair with Applied Biosystem (ABI) 3500 XL sequencer (Deng et al., 2015). Use of M13 universal primer pair resolved the problem where errors usually occur near the beginning and end of the sequencing run. It also abolishes the needs of internal primers to fill the gap sequences as performed by Piaggio et al. (2012). However, the invention of Next-Generation Sequencing renders this method less cost-effective in case of a large number of targets. Nevertheless, it is still preferable to conduct partial genome sequencing of influenza viruses as it can generate a longer sequence read.

\section{Next-Generation sequencing (NGS)}

Next-Generation sequencing (NGS) refers to newer DNA sequencing technologies which enable speedy, highthroughput sequencing. A large number of genome sequences can be generated concurrently, significantly reduce the cost of sequencing compared to traditional Sanger method. Nowadays, many NGS platforms are available and usually manufacturer specific. Roche 454 Life Sciences developed the first NGS instrument with pyrosequencing. Pyrosequencing is a sequencing-bysynthesis method, driven by four types of the enzyme to measure pyrophosphate release during incorporation of dNTP to the complementary template strand. One of the four dNTPs are added sequentially; the light will only be detected when pairing with the template occurs. Therefore, the sequence of the new strand is deduced from the order of dNTP incorporated into the growing strand. Roche 454 pyrosequencing was used to identify amino acid deletions in NA stalk of LPAI viruses (subtype H6N1) between two avian hosts (ducks and turkeys) in poultry, implying viruses adaptation in turkey host and change of tissue tropism from gastrointestinal tract to respiratory tract through NA deletion (Croville et al., 2012). Biotage PSQ 96MA platform pyrosequencer was employed to identify antiviral resistance markers in $\mathrm{M} 2$ protein and NA protein of H5N1 virus (Deyde et al., 2009). More recently, Wang et al. (2018) have developed a combined RT-PCR and pyrosequencing method to identify serine at the P6 position of HACS of $\mathrm{H} 5 \mathrm{~N} 1$ virus, which is a marker of high pathogenicity (Wang et al., 2018). Illumina's NGS platform uses a different sequenceby-synthesis approach in which enzyme-driven processes and sequencing take place in a flow cell. DNA molecules are fragmented and modified before loading into the flow cell. The oligonucleotides coated on the flow cell serve as primers to generate colonies of the modified DNA through bridge amplification. The colonies are then sequenced using fluorescently labelled-dNTPs, which are incorporated into a growing strand in a reversible termination manner (Buermans and den Dunnen, 2014).

Illumina has launched different sequencing instruments such as miSeq, NextSeq 500, HiSeq2500, and HiSeq $X$ Ten, producing a different number of read per run and different read length to fit different demands. Illumina miSeq was combined with nanomicroarray, being developed as a new diagnostic platform capable of detecting and subtyping influenza viruses as well as to characterize the whole genome sequence (Zhao et al., 2015). Other NGS platforms, such as Ion Torrent Personal Genome Machine (PGM)/Photon, SOLiD (sequencing by ligation) and Pacific Biosciences Singlemolecule real-time (SMRT) sequencing are available. Each platform has utilized different sequencing technique and has its own merits and demerits. Most of the whole genome-sequenced influenza virus has employed the Roche 454, Illumina, and Ion Torrent PGM/Photon platform (Ali et al., 2016). NGS enables large scale diagnosis, and sequence information can be obtained simultaneously. Hence, not only highly virulence strain of viruses can be identified, but the emergence of a new strain with pandemic potential can also be predicted from the sequences. In spite of the advanced robust property of NGS, the equipment is very costly. Constant maintenance is also required, making it unaffordable in many diagnostic laboratories.

\section{Rapid influenza diagnostic test (RIDTs)}

Rapid influenza diagnostic test (RIDTs) are immunoassays, aim to identify the presence of influenza $A$ or B virus by detecting viral NP antigen. They are also known as point-of-care (POC) tests in which results can be obtained within 30 minutes that available in clinical settings. Most of the RIDTs employ immunochromatographic (ICG) technique (also known as lateral flow), to detect the presence of viruses using highly sensitive monoclonal antibodies. RIDTs are available in dipstick, housed cassette or card format. The ICG test comprises a sample pad, a conjugate pad, nitrocellulose (NC) membrane, and an absorption pad, all capable of transporting fluid spontaneously. When a sample contains the target analyte is applied to the sample pad, it will be absorbed and migrates to the conjugate pad via capillary action, releasing the immobilized tag conjugated-antiviral antibodies. These antibodies will bind to the target antigens, forming complexes that continue to flow with unreacted antibodies to the NC membrane that contains 
antiviral monoclonal antibodies and anti-immunoglobulin $\mathrm{G}(\mathrm{lg} G)$ in well-demarcated areas. The antigen-antibody complexes and unreacted antibodies will be captured by the antigen-specific antibodies and anti-lgG, respectively, producing two visible lines that indicate positive test result and correct functioning of the test (Dziabowska et al., 2018). RIDTs available in the market can detect either IFAV only or both IFAV and influenza B viruses (IFBV) with or without discrimination between $A$ and $B$ viruses (Vemula et al., 2016). For example, NOW Influenza A \& B Test (Binax), Directigen Flu A+B (Becton Dickinson), Quick Vue Influenza $A+B$ (Quidel) can detect and differentiate between A \& B. Most of the RIDTs are meant for general detection of all IFAVs (explicitly optimized for seasonal influenza viruses), by targeting NP that is conserved between different strains of IFAV. The effectiveness of RIDTs in detecting AIV was evaluated in some studies. A study had evaluated the performance of two RIDTs (Directigen Flu $A+B$ and X/pect Flu $A+B$ kit (Thermo Scientific) relative to shell vial assay in detecting AIV subtype H5N1 and H9N2, indicating the tests might capable to detect AIV but are not readily sensitive for wild type strains of the viruses compared to those coldadapted one (Fedorko et al., 2006). This may imply these tests are insensitive in detecting AIV if poultry attacked by newly introduced wild type strain. Another study tested the efficiency of five RIDTs (Directigen Flu A, QuickVue Influenza test kit, FLU OIA (ThermoBiostar), Zstat Flu (ZymeTx, Inc.) and NOW FLU A Test) in detecting AIV of low pathogenicity (subtype H6N2) showed unsatisfied results (Directigen and Flu OIA only detected 2/39 individual oropharyngeal swabs and NOW FLU A detected 1/39 compared to virus isolation method (Woolcock and Cardona, 2005). However, a study performed by Sakai-Tagawa et al. (2014) indicated most of the commercially available RIDTs in Japan capable of detecting AIV (only subtype H5N1 and H7N9 tested) with different detection sensitivity (Sakai-Tagawa et al., 2014). Therefore, the suitability of RIDTs in diagnostic field investigations is controversial. RIDTs meant for AIV are also available currently, such as Anigen Rapid AIV Ag Test kit (Bionote), Poctem Avian Influenza kit (Sysmex, Co.) for general detection of AIV of all subtypes, Anigen Rapid H5 AIV Ag Test kit (Bionote) specifically for the $\mathrm{H} 5 \mathrm{~N} 1$ virus. However, reported the sensitivity of Anigen Rapid AIV Ag Test kit (Bionote) and Anigen Rapid AIV Ag Test kit (Bionote) compared with RT-PCR assay in testing field samples were $50 \%$ and $25 \%$ respectively (Wibowo et al., 2017). Another study evaluated Anigen Rapid AIV Ag Test kit for detecting HPAI H5N1 virus in Vietnam using 175 samples had reported diagnostic test sensitivity of $61 \%$ and specificity of $98 \%$ relative to qRT-PCR (Loth et al., 2015). Limited sensitivity of these RIDTs retreats their application in most diagnostic laboratories. However, the reported sensitivity of Sysmex Avian Influenza kit tested with laboratory test samples was $71 \%$ relative to virus isolation method and $92 \%$ relative to RT-PCR when tested field samples.

Furthermore, this kit was tested to be highly specific to AIV and was recommended to be applied in the field testing (Ibrahim and Ikuta, 2011). The virus titre present in samples often influence the sensitivity of RIDTs. Types of sample collected is also another factor renders RIDTs less sensitive as the tissue preferences of viral shedding may different for different species of hosts. In spite of the issue of limited sensitivity, ease of use, and rapid results offered by RIDTs making it suitable to be used for screening suspected samples before laboratory confirmation.

\section{NEW DIAGNOSTIC APPROACHES}

\section{Nucleic acid sequencing-based amplification (NASBA)}

Nucleic acid sequencing-based amplification (NASBA) is a method which amplifies RNA molecule with three enzymes, avian myeloblastosis virus reverse transcriptase (AMV-RT), T7 RNA polymerase and RNase $\mathrm{H}$, and two primers. The first primer is used to produce a complementary sequence of the RNA template by AMV$\mathrm{RT}$. When RNase $\mathrm{H}$ degrades the RNA template, the second primer with T7 promoter sequence on $5^{\prime}$ end anneals to the complementary strand, extended by AMV$\mathrm{RT}$, producing double-stranded DNA with T7 promoter sequence. T7 RNA polymerase docks to the DNA at the promoter region transcribes RNA copy. Each new RNA will re-enter the process of first strand synthesis, RNA degradation, second strand synthesis, and RNA transcription until primers deplete. Amplified RNA molecules are then detected utilizing electrochemiluminescence (ECL), using a capture probe, and ruthenium-based ECL tagged detection probe. Collins et al. (2003b) used this method to detect LPAI and HPAI viruses of subtype $\mathrm{H} 5$ by targeting HACS (Collins et al., 2003b). Another NASBA assay for detection AIV subtype $\mathrm{H} 7$ was conducted by the same group, reported a limit of detection of $10^{5}$-fold dilution of template, but diagnostic sensitivity and specificity of the assay were not performed in two studies (Collins et al., 2003a). Lau et al. (2004) developed NASBA-AIV assay for detection of all subtypes of AIV by targeting the highly conserved $\mathrm{M}$ gene with an analytical sensitivity of $10^{2.92-3.92} \mathrm{EID}_{50} / \mathrm{mL}$ (median egg infectious dose) (Lau et al., 2004). Moore et al. (2010) developed a commercial real-time NASBA assay, which introduced a molecular beacon label with fluorescent dye and a quencher into reaction mixtures, to detect H5N1 virus and was evaluated using 19 clinical samples obtained from confirmed human cases of H5N1 virus infection in China, with analytical sensitivity of 0.01 $\mathrm{TCID}_{50}$ (median tissue culture infectious dose) and 10 copies RNA/ $\mu \mathrm{L}$ and diagnostic sensitivity of $100 \%$ (Moore et al., 2010; Dziabowska et al., 2018). Another study which compared NASBA assay with viral isolation method for detection of the H5N1 virus, reporting the sensitivity of $75.5 \%$ (Shan et al., 2003). NASBA assay is very suitable for AIVs screening since AIVs are RNA virus. CDNA synthesis is not required for amplification of the target gene. Despite high sensitivity offered by NASBA assay, high cost of the commercial kit and longer TAT compared to qRT-PCR renders it less practical in screening AIV. 
Loop-mediated isothermal amplification-based assay (LAMP)

Loop-mediated isothermal amplification-based assay (LAMP) was developed by Notomo et al. (2000), using at least four different primers to recognize six distinct regions of target gene and DNA polymerase with strand displacement activity, leading to the formation of many stem-loop DNA at a constant temperature between 60-65 ${ }^{\circ} \mathrm{C}$. Positive amplification is detected by the formation of turbid solution as a result of magnesium pyrophosphate precipitate release during amplification, or by adding intercalating dye (SYBR green) which bind to stem-loop DNA, forming a yellow-green colour solution under UV light source. Accumulation of the LAMP product also can be detected in real time using an instrument. RT-LAMP is specifically for RNA detection by incorporating RT (Notomi et al., 2000). RT-LAMP based assays have been successfully developed for detection of AIV subtype $\mathrm{H} 5 \mathrm{~N} 1, \mathrm{H} 7 \mathrm{~N} 9, \mathrm{H} 3$ and $\mathrm{H} 10$ with reported sensitivity equal or higher than RT-PCR or virus isolation method (Imai et al., 2006; Jayawardena et al., 2007; Peng et al., 2011; Bao et al., 2014; Luo et al., 2015). Detection of RT-LAMP products with lateral flow device was also developed for the detection of H7N9 virus (Ge et al., 2013). However, two commercial LAMP for detection of AIV H5 and $\mathrm{H} 7$ subtype were shown to exhibit reduced sensitivity compared to the qRT-PCR method, claiming primer mismatches with phylogenetically different AIV isolates used in the study was the potential cause of the result (Postel et al., 2010). Hence, designed primers should target highly conserved regions irrespective of viral lineages for broad-spectrum detection and be continuously reviewed to maintain sensitivity. RT-LAMP assay is very suitable to be used in a resource-limited setting, particularly useful in field testing of AIV as this method does not need sophisticated equipment, only thermal block or water bath set at a constant temperature as well as a UV illuminator are required.

\section{DNA microarray}

DNA microarray is a collection of probes or oligonucleotides immobilized on a solid surface, for analyzing or measuring gene expression. Each spot of the array contains known gene sequences. This feature enables simultaneous screening of thousands of genes in a single reaction at different loci. The principle of microarray is based on the hybridization of two complementary strands. Messenger RNA (mRNA) isolated from the sample are reverse transcribed into cDNA tagged by fluorescence dye (Cy3 or Cy5). The fluorescently labelled cDNAs are subjected to hybridize to its complementary probes in the array. After washing unbound sequences, the array is scanned by a laser beam which excites fluorophores of hybridized sequences, emitting fluorescence signals that could be captured by a camera. FluChip-55 is a microarray employs 55 pairs of capture-label probes, targeting $M, H A$ and NA gene of IFAV subtype $\mathrm{H} 1 \mathrm{~N} 1, \mathrm{H} 3 \mathrm{~N} 2$, and $\mathrm{H} 5 \mathrm{~N} 1$, $M, N P$ and $H A$ gene of IFBV, used to differentiate $A$ and $B$ virus and subtyping IFAV (Townsend et al., 2006). Whereas, MChip utilizes 15 capture probes correspond to the highly conserved regions of $\mathrm{M}$ gene, to detect a wide range of any subtypes or a particular subtype of IFAV by analyzing the distinctive pattern of fluorescence signals intensity exhibited by different subtypes of the viruses (Dawson et al., 2006). It had been demonstrated capable of identifying IFAV of H5N1 subtype from different hosts as well as subtype $\mathrm{H} 1 \mathrm{~N} 1$ and $\mathrm{H} 3 \mathrm{~N} 2$ with a sensitivity of 97\% (Dawson et al., 2007). Nanochip 400 system, which uses one probe for the $\mathrm{M}$ gene and 97 probes for HACS was shown to be useful in detecting HPAl viruses of H5 and $\mathrm{H} 7$ (Gall et al., 2009). In 2010, Zhao et al. (2010) reported a novel microarray platform which employed gold nanoparticle (AuNP) coupled with silver staining to detect $\mathrm{H} 5 \mathrm{~N} 1$ virus and distinguish from the other subtypes such as $\mathrm{H} 1 \mathrm{~N} 1$ and $\mathrm{H} 3 \mathrm{~N} 2$ in the absence of RT-PCR (Zhao et al., 2010). cDNA microarray for detection and subtyping AIV $(\mathrm{H} 5, \mathrm{H} 7$, and $\mathrm{H} 9)$ was reported with a sensitivity of $70 \%$ (Maughan et al., 2014). Microarray is a powerful method that enables high-throughput screening. However, the requirement of sophisticated equipment and trained person for data collection and analysis have restricted its application in AIV detection and surveillance.

\section{Biosensor}

A biosensor is an analytical device which integrates biological recognition element (also known as bioreceptor) with a physiochemical transducer. It relies on the interaction between the target analyte and bioreceptor, in which the interaction is transduced into quantitative signals that correspond to the concentration of the target. Bioreceptors could be either antibodies, aptamers DNA, or RNA molecules, which only recognize the molecules of interest. The biosensor can be further characterized based on the principle of signal transduction integrated, such as piezoelectric, optical, and electrochemical biosensors. Therefore, biosensors are usually named following the bioreceptor and transduction element used. In piezoelectric biosensor, crystals are sandwiched between two electrodes. Analyte-bioreceptor interaction causes the generation of piezoelectricity by the crystals, result changes in resonant frequency, which will be measured. Quartz crystal microbalance (QCM) is frequently used in a piezoelectric biosensor. $\mathrm{Li}$ et al. (2011) developed a QCM immunosensor which combined polyclonal antibodies against $\mathrm{HA}$ of $\mathrm{H} 5 \mathrm{~N} 1$ virus that are immobilized on the gold surface of the QCM crystal through self-assembled monolayer (SAM) of 16mercaptohexadecanoic acid (MHDA) to capture viruses and magnetic nanobeads coated with anti-H5 antibodies to amplify signal with detection limit of $0.0128 \mathrm{HAU}$ ( $\mathrm{Li}$ et al., 2011). Another study had replaced antibodies with aptamers, short single-stranded oligonucleotides that bind specifically with viruses, reported a detection limit of 1 HAU but shorter detection time of $1 \mathrm{~h}$ compared to immunosensor which required $2 \mathrm{~h}$ as described 
previously (Brockman et al., 2013). The higher detection limit was attributed to the failure of aptamer coated nanobeads as a signal amplifier. More recently, Wang et al. (2017) had developed a label-free, nanowell-based QCM aptasensor, which used nanoporous gold film to form a nanowell-based electrode, which in turn immobilized with aptamers to detect H5N1 virus (Wang et al., 2017). Some other QCM aptasensors which used hydrogel and $\mathrm{N}$-acetylglucosamine were also reported (Wang and Li, 2013; Wang and Li, 2016). In the case of optical biosensors, the specific interaction of bioreceptors and target molecules is visualized utilizing surface plasmon resonance (SPR), absorption, luminescence, or fluorescence. For instances, SPR biosensor measures the refractive index $(\mathrm{RI})$ changes caused by the aptamervirus complex. Aptamers are immobilized on sensor gold surface via streptavidin-biotin binding. Binding of viruses to aptamers on the sensor surface changes the RI, in turn resulting changes in the SPR angle. Bai et al. (2012) used Spreeta SPR detector (Texas Instruments, Dallas, TX USA) to develop a portable SPR aptasensor that specifically detect $\mathrm{H} 5 \mathrm{~N} 1$ virus in $1.5 \mathrm{~h}$ with a detection limit of 0.128 HAU (Bai et al., 2012). A fluorescence biosensor which used luminescent cadmium telluride (CdTe) quantum dots to detect H5N1 virus was reported. This biosensor measures photoluminescence intensity emitted by quantum dots, which is sensitive to $\mathrm{pH}$ changes. Chromophore with $F_{0}$ base piece and $F_{1}$ head group of ATPase ( $\left.F_{0} F_{1}-A T P a s e\right)$ and antibody of $\beta$ subunit, attached with CdTe quantum dots form the core part of the biosensor. The outer part consists of antibody which linked to the core part by streptavidin-biotin binding. Binding of virus to the antibody affects $\mathrm{H}^{+}$which are produced during $\mathrm{F}_{0} \mathrm{~F}_{1}$-ATPase catalyzed the conversion of ATP from adenosine diphosphate (ADP), to be pumped out from the chromophore, which subsequently influences photoluminescence intensity of quantum dots (Nguyen et al., 2012). Another fluorescence aptasensor developed by Pang et al. (2015) utilized thiazole orange (TO) for fluorescence sensing of $\mathrm{Al} \mathrm{H} 5 \mathrm{~N} 1$ virus in solution. $\mathrm{Ag} @ \mathrm{SiO}_{2}$ core-shell nanoparticles were coated with $\mathrm{HA}$ specific aptamers. After interacting with the HA protein of the virus, the aptamers transform into a G-quadruplex that could capture free TO in the solution. $\mathrm{Ag} @ \mathrm{SiO}_{2}$ core-shell nanoparticles enhanced fluorescence signal emitted by TO using SPR. This detection method took only 30 minutes to complete and was demonstrated to detect HA protein of $\mathrm{H} 5 \mathrm{~N} 1$ virus in aqueous buffer and human serum with a detection limit of 2 and $3.5 \mathrm{ng} / \mathrm{mL}$ respectively (Pang et al., 2015). The third type of biosensor based on signal transduction is electrochemical biosensors, which identify target analyte by changes in the electrical properties due to biochemical reactions. Electrochemical biosensors can be further characterized based on electrical parameter measured, such as amperometric, impedimetric, voltammetric, potentiometric, etc. For instances, impedance biosensors measure electrical resistance caused by the bioreceptor-analyte complexes by imposing a constant sinusoidal voltage. Lum et al. (2015) had developed an impedance aptasensor which integrated a microfluidics flow cell and an interdigitated microelectrode for specific detection $\mathrm{H} 5 \mathrm{~N} 1$ virus with a detection limit of $0.0128 \mathrm{HAU}$ (Lum et al., 2015). A more comprehensive review of biosensors for detection AIVs was reported by Grabowska et al. (2014) and Wang and Li (2016) (Grabowska et al., 2014; Wang and $\mathrm{Li}, 2016)$. Biosensors for AIVs detection are not readily applied currently. But, they could be potentially, fully utilized in the future as they are more cost-effective and portable to be used in field testing.

\section{Smartphone-based fluorescent diagnostic system}

Smartphones are one of the ubiquitous gadgets in this era of technology. The built-in camera on smartphone can be utilized for the detection of AIV. Yeo et al. (2016) had developed a smartphone-based fluorescent diagnostic system for HPAI H5N1 viruses with a sensitivity of $96.55 \%$ and specificity of $98.55 \%$ (Yeo et al., 2016a). This diagnostic system was evaluated with H9N2 virus with a reported sensitivity of $94.4 \%$ and $95.23 \%$ (relative to qRTPCR) for oropharyngeal and cloacal samples, respectively (Yeo et al., 2016b). This platform comprises a fluorescent ICG test strip that used latex beads conjugated with coumarin-derived dendrimer and anti-NP antibodies and a smartphone-based fluorescence detector. When a fluorescent lateral flow strip is inserted into the measurement module of the detector which is protected from the external light source, LED light excites coumarin-derived dendrimer, fluorescence emitted are collected and detected by the smartphone camera module. A software application was used to estimate total fluorescence intensities by averaging the pixel intensity values. The result is reported as the ratio of fluorescence intensity from the test line to control line of the strip and could be sent as soon as possible via wireless communication to the database (Yeo et al., 2016a). This diagnostic system is very suitable for use in a resourcelimited setting, such as field-testing in which expensive instrument like fluorescence microscope is not needed. Furthermore, this method exhibits higher sensitivity compared to conventional RIDTs. Hence, the development of a user-friendly measurement application is required to perform the test.

\section{CONCLUSION}

Diagnostic and surveillance of $\mathrm{Al}$ are challenging. Constant genetic mutation of AIV through antigenic drift or antigenic shift often causes outbreaks in poultry, which pose a risk in food supply and result in significant economic loss. Migratory birds are also another source of mutation in poultry. LPAl viruses shedding in poultry is often asymptomatic or exhibits mild clinical symptoms, which makes them escape detection, is a potential source of the outbreak. Selection of detection methods is based on the ease to use onsite, speed, and sensitivity. From all the Al detection methods mentioned in this article, RTPCR combines with amplicons sequencing is the most favourable method due to high sensitivity and accuracy 
offered despite longer TAT. However, a sensitive, costeffective, and reliable method to be available in poultry farms, that can be performed by farmers or workers without requiring technical skills is preferred to manage outbreaks. Also, LPAI viruses are often left behind, but their influence should be taken into consideration. New detection approaches that offer portability, high sensitivity, and accuracy, are potentially adapted in farms in the future as it could save the time of samples transferring and provide more rapid result. Hence, comprehensive research to further expand the detection range and evaluation study are required to ensure their reliability.

\section{ACKNOWLEDGEMENTS}

We would like to acknowledge the financial support given by Transdisciplinary Research Grant Scheme 203/PPSK/6763002.

\section{REFERENCES}

Ali, R., Blackburn, R. M. and Kozlakidis, Z. (2016). Next-Generation sequencing and influenza virus: A short review of the published implementation attempts. HAYATI Journal of Biosciences 23(4), 155159.

Alvarez, A. C., Brunck, M. E., Boyd, V., Lai, R., Virtue, E., Chen, W., Bletchly, C., Heine, H. G. and Barnard, R. (2008). A broad spectrum, one-step reverse-transcription PCR amplification of the Neuraminidase gene from multiple subtypes of influenza A virus. Virology Journal 5, 77.

Aminoff, D. (1961). Methods for the quantitative estimation of $\mathrm{N}$-acetylneuraminic acid and their application to hydrolysates of sialomucoids. The Biochemical Journal 81(2), 384-392.

Bai, H., Wang, R., Hargis, B., Lu, H. and Li, Y. (2012). A SPR aptasensor for detection of avian influenza virus H5N1. Sensors (Basel) 12(9), 12506-12518.

Bao, H., Zhao, Y., Wang, Y., Xu, X., Shi, J., Zeng, X., Wang, $X$. and Chen, H. (2014). Development of a reverse transcription loop-mediated isothermal amplification method for the rapid detection of subtype H7N9 avian influenza virus. BioMed Research International 2014, 1-8.

Brockman, L., Wang, R., Lum, J. and Li, Y. (2013). QCM aptasensor for rapid and specific detection of avian influenza virus. Journal of Applied Biosensor 02(04), 97-103.

Brown, J. D., Stallknecht, D. E., Berghaus, R. D., Luttrell, M. P., Velek, K., Kistler, W., Costa, T., Yabsley, M. J. and Swayne, D. (2009). Evaluation of a commercial blocking enzyme-linked immunosorbent assay to detect avian influenza virus antibodies in multiple experimentally infected avian species. Clinical and Vaccine Immunology, 16(6), 824-829.

Buermans, H. P. and den Dunnen, J. T. (2014). Next Generation sequencing technology: Advances and applications. Biochimica et Biophysica Acta 1842(10), 1932-1941.
Butt, K. M., Smith, G. J. D., Chen, H., Zhang, L. J., Leung, Y. H. C., Xu, K. M., Lim, W., Webster, R. G., Yuen, K. Y., Peiris, J. S. M. and Guan, Y. (2005). Human infection with an avian H9N2 influenza A virus in Hong Kong in 2003. Journal of Clinical Microbiology, 43(11), 5760-5767.

CDC. (2018). Asian Lineage Avian Influenza A (H7N9) Virus. [Online] Available from: https://www.cdc.gov/flu/avianflu/h7n9-virus.htm [Accessed 26 Nov 2018].

Chaharaein, B., Omar, A. R., Aini, I., Yusoff, K. and Hassan, S. S. (2009). Detection of H5, H7 and H9 Subtypes of Avian Influenza Viruses by Multiplex Reverse Transcription-Polymerase Chain Reaction. Microbiological Research 164(2), 174-179.

Collins, R. A., Ko, L. S., Fung, K. Y., Chan, K. Y., Xing, J., Lau, L. T. and Yu, A. C. (2003a). Rapid and sensitive detection of avian influenza virus subtype $\mathrm{H} 7$ using NASBA. Biochemical Biophysical Research Communications 300(2), 507-515.

Collins, R. A., Ko, L. S., So, K. L., Ellis, T., Lau, L. T. and Yu, A. C. (2003b). A NASBA method to detect high- and low-pathogenicity $\mathrm{H} 5$ avian influenza viruses. Avian Diseases 47(3 Suppl), 1069-1074.

Coons, A. H., Creech, H. J. and Jones, R. N. (1941). Immunological properties of an antibody containing a fluorescent group. Proceedings of the Society for Experimental Biology and Medicine 47(2), 200-202.

Croville, G., Soubies, S. M., Barbieri, J., Klopp, C., Mariette, J., Bouchez, O., Camus-Bouclainville, C. and Guérin, J. L. (2012). Field monitoring of avian influenza viruses: Whole-genome sequencing and tracking of neuraminidase evolution using 454 pyrosequencing. Journal of Clinical Microbiology 50(9), 2881-2887.

Dawson, E. D., Moore, C. L., Dankbar, D. M., Mehlmann, M., Townsend, M. B., Smagala, J. A., Smith, C. B., Cox, N. J., Kuchta, R. D. and Rowlen, K. L. (2007). Identification of $A / H 5 N 1$ influenza viruses using a single gene diagnostic microarray. Analytical Chemistry 79(1), 378-384.

Dawson, E. D., Moore, C. L., Smagala, J. A., Dankbar, D. M., MehImann, M., Townsend, M. B., Smith, C. B., Cox, N. J., Kuchta, R. D. and Rowlen, K. L. (2006). MChip: A tool for influenza surveillance. Analytical Chemistry 78(22), 7610-7615.

Deng, Y. M., Spirason, N., lannello, P., Jelley, L., Lau, H. and Barr, I. G. (2015). A simplified sanger sequencing method for full genome sequencing of multiple subtypes of human influenza $A$ viruses. Journal of Clinical Virology 68, 43-48.

Deyde, V. M., Nguyen, T., Bright, R. A., Balish, A., Shu, B., Lindstrom, S., Klimov, A. I. and Gubareva, L. V. (2009). Detection of molecular markers of antiviral resistance in influenza $A(\mathrm{H} 5 \mathrm{~N} 1)$ viruses using a pyrosequencing method. Antimicrobial Agents and Chemotherapy 53(3), 1039-1047.

Dziabowska, K., Czaczyk, E. and Nidzworski, D. (2018). Detection methods of human and animal 
influenza virus-current trends. Biosensors (Basel) 8(4).

Eisfeld, A. J., Neumann, G. and Kawaoka, Y. (2014). Influenza A virus isolation, culture and identification. Nature Protocols 9(11), 2663-2681.

Fedorko, D. P., Nelson, N. A., McAuliffe, J. M. and Subbarao, K. (2006). Performance of rapid tests for detection of avian influenza $A$ virus types $\mathrm{H} 5 \mathrm{~N} 1$ and H9N2. Journal of Clinical Microbiology 44(4), 15961597.

Gall, A., Hoffmann, B., Harder, T., Grund, C., Höper, D. and Beer, M. (2009). Design and validation of a microarray for detection, hemagglutinin subtyping, and pathotyping of avian influenza viruses. Journal of Clinical Microbiology 47(2), 327-334.

Ge, Y., Wu, B., Qi, X., Zhao, K., Guo, X., Zhu, Y., Qi, Y., Shi, Z., Zhou, M., Wang, H. and Cui, L. (2013). Rapid and sensitive detection of novel avian-origin influenza A (H7N9) virus by reverse transcription loopmediated isothermal amplification combined with a lateral-flow device. PLOS ONE 8(8), e69941.

Grabowska, I., Malecka, K., Jarocka, U., Radecki, J. and Radecka, H. (2014). Electrochemical biosensors for detection of avian influenza virus-current status and future trends. Acta Biochimica Polonica, 61(3), 471-478.

Ibrahim, M. and Ikuta, K. (2011). Field diagnosis of avian influenza H5N1 virus in Egypt using RAPID immunochromatographic diagnostic tests. International Journal of Virology 7(4), 184-190.

Imai, M., Ninomiya, A., Minekawa, H., Notomi, T., Ishizaki, T., Tashiro, M. and Odagiri, T. (2006). Development of H5-RT-LAMP (loop-mediated isothermal amplification) system for rapid diagnosis of H5 avian influenza virus infection. Vaccine 24(44), 6679-6682.

Jayawardena, S., Cheung, C. Y., Barr, I., Chan, K. H., Chen, H., Guan, Y., Peiris, J. S. M. and Poon, L. L. M. (2007). Loop-mediated isothermal amplification for influenza A (H5N1) virus. Emerging Infectious Diseases 13(6), 899-901.

Jiao, P., Song, Y., Huang, J., Xiang, C., Cui, J., Wu, S., Qu, N., Wang, N., Ouyang, G. and Liao, M. (2018). H7N9 avian influenza virus is efficiently transmissible and induces an antibody response in chickens. Frontiers in Immunology 9, 789-789.

Killian, M. L. (2008). Hemagglutination assay for the avian influenza virus. In: Avian Influenza Virus. Spackman, E. (ed.). Humana Press. pp 47-52.

Klimov, A., Balish, A., Veguilla, V., Sun, H., Schiffer, J., Lu, X., M Katz, J. and Hancock, K. (2012). Influenza virus titration, antigenic characterization, and serological methods for antibody detection. In: Influenza Virus. Methods in Molecular Biology (Methods and Protocols), vol 865. Kawaoka Y., Neumann G. (eds). Humana Press. pp 25-51.

Krammer, F., Smith, G. J. D., Fouchier, R. A. M., Peiris, M., Kedzierska, K., Doherty, P. C., Palese, P., Shaw, M. L., Treanor, J., Webster, R. G. and
García-Sastre, A. (2018). Influenza. Nature Reviews Disease Primers 4(1), 3.

Krauss, S., Walker, D. and Webster, R. G. (2012). Influenza virus isolation. Methods in Molecular Biology 865, 11-24.

Lau, L. T., Banks, J., Aherne, R., Brown, I. H., Dillon, N., Collins, R. A., Chan, K. Y., Fung, Y. W., Xing, J. and Yu, A. C. (2004). Nucleic acid sequence-based amplification methods to detect avian influenza virus. Biochemical and Biophysical Research Communications 313(2), 336-342.

Leang, S. K. and Hurt, A. C. (2017). Fluorescencebased neuraminidase inhibition assay to assess the susceptibility of influenza viruses to the neuraminidase inhibitor class of antivirals. Journal of Visualized Experiments 122, 55570.

Lee, M. S., Chang, P. C., Shien, J. H., Cheng, M. C. and Shieh, H. K. (2001). Identification and subtyping of avian influenza viruses by reverse transcription-PCR. Journal of Virology Methods 97(1-2), 13-22.

Li, D., Wang, J., Wang, R., Li, Y., Abi-Ghanem, D., Berghman, L., Hargis, B. and Lu, H. (2011). A Nanobeads amplified QCM immunosensor for the detection of avian influenza virus H5N1. Biosensors and Bioelectronics 26(10), 4146-4154.

Lin, Y., Gu, Y. and McCauley, J. W. (2016). Optimization of a quantitative micro-neutralization assay. Journal of Visualized Experiments (118), 54897.

Liu, C. (1955). Studies on influenza infection in Ferrets by means of fluorescein-labelled antibody. The Journal of Experimental Medicine 101(6), 665.

Loth, L., Long, P. T., Tung, N., Dang, N. H. and Newman, S. (2015). Test characteristics of the Anigen ${ }^{\circledR}$ Rapid AIV Ag Test (Avian Influenza Type A Rapid Antigen Test) in Ducks in Vietnam. Virology \& Mycology 4(1).

Lum, J., Wang, R., Hargis, B., Tung, S., Bottje, W., Lu, H. and Li, Y. (2015). An impedance aptasensor with microfluidic chips for specific detection of $\mathrm{H} 5 \mathrm{~N} 1$ avian influenza virus. Sensors (Basel) 15(8), 18565-18578.

Luo, S., Xie, Z., Xie, L., Liu, J., Xie, Z., Deng, X., Huang, L., Huang, J., Zeng, T. and Khan, M. I. (2015). Reverse-transcription, loop-mediated isothermal amplification assay for the sensitive and rapid detection of $\mathrm{H} 10$ subtype avian influenza viruses. Virology Journal 12(1), 145.

Maughan, M. N., Bliss, T. W., Chung, I., Suarez, D. L. and Keeler JR, C. L. (2014). Detection and identification of avian influenza virus by cona microarray. Journal of Microbial and Biochemical Technology S2: 005.

Mehdi Hadipour, M. (2009). Detection of avian influenza virus antigen in chicken tissues following intranasal inoculation. Asian Journal of Animal and Veterinary Advances 4(2), 93-98.

Monne, I., Ormelli, S., Salviato, A., De Battisti, C., Bettini, F., Salomoni, A., Drago, A., Zecchin, B., Capua, I. and Cattoli, G. (2008). Development and validation of a one-step real-time $\mathrm{PCR}$ assay for simultaneous detection of subtype $\mathrm{H} 5, \mathrm{H} 7$, and $\mathrm{H} 9$ 
avian influenza viruses. Journal of Clinical Microbiology 46(5), 1769-1773.

Moore, C., Telles, J. N., Corden, S., Gao, R. B., Vernet, G., Van Aarle, P. and Shu, Y. L. (2010). Development and validation of a commercial real-time nasba assay for the rapid confirmation of influenza $A$ H5N1 virus in clinical samples. Journal of Virology Methods 170(1-2), 173-176.

Nguyen, T. H., Ung, T. D. T., Vu, T. H., Tran, T. K. C. Dong, V. Q., Dinh, D. K. and Nguyen, Q. L. (2012). Fluorescence biosensor based on CdTe quantum dots for specific detection of $\mathrm{H} 5 \mathrm{~N} 1$ avian influenza virus. Advances in Natural Sciences: Nanoscience and Nanotechnology 3(3), 035014.

Notomi, T., Okayama, H., Masubuchi, H., Yonekawa, T., Watanabe, K., Amino, N. and Hase, T. (2000). Loop-mediated isothermal amplification of DNA. Nucleic Acids Research, 28(12), E63-E63.

Nur Adibah, M., Zailina, H. and Arshad, S. S. (2017). Avian Influenza Outbreaks in Malaysia, 1980 - 2017. Asia Pacific Environmental and Occupational Health Journal 3(2), 1-14.

OIE. (2015). Chapter 10. 4. Infection with avian influenza viruses. [Online] Available from: https://www.oie.int/fileadmin/Home/eng/Health_standa rds/tahc/current/chapitre_avian_influenza_viruses.pdf [Accessed 18 Sept 2019]

OIE. (2018). Situation report for highly pathogenic avian influenza [Online] Available from:http://www.oie.int/fileadmin/Home/eng/Animal_H ealth in the World/docs/pdf/OIE Al situation_report/ OIE_SituationReport_Al_August2018.pdf [Accessed 27 Nov 2018]

Pang, Y., Rong, Z., Wang, J., Xiao, R. and Wang, S. (2015). A fluorescent aptasensor for H5N1 influenza virus detection based-on the core-shell nanoparticles metal-enhanced fluorescence (MEF). Biosensor and Bioelectronics 66C, 527-532.

Pedersen, J. C. (2008). Neuraminidase-inhibition assay for the identification of influenza a virus neuraminidase subtype or neuraminidase antibody specificity. In: Avian Influenza Virus. Methods in Molecular Biology ${ }^{\mathrm{TM}}$, vol 436. Spackman, E. (ed.). Humana Press. pp 67-75.

Peng, Y., Xie, Z., Liu, J., Pang, Y., Deng, X., Xie, Z., Xie, L., Fan, Q., Feng, J. and Khan, M. I. (2011). Visual detection of $\mathrm{H} 3$ subtype avian influenza viruses by reverse transcription loop-mediated isothermal amplification assay. Virology Journal 8, 337.

Piaggio, A. J., Shriner, S. A., VanDalen, K. K., Franklin, A. B., Anderson, T. D. and Kolokotronis, S. O. (2012). Molecular surveillance of low pathogenic avian influenza viruses in wild birds across the United States: Inferences from the hemagglutinin gene. PLoS One 7(12), e50834.

Poovorawan, Y., Pyungporn, S., Prachayangprecha, S. and Makkoch, J. (2013). Global alert to avian influenza virus infection: From H5N1 to H7N9. Pathogens and Global Health 107(5), 217-223.
Postel, A., Letzel, T., Frischmann, S., Grund, C., Beer, M. and Harder, T. (2010). Evaluation of two commercial loop-mediated isothermal amplification assays for detection of avian influenza $\mathrm{H} 5$ and $\mathrm{H} 7$ hemagglutinin genes. Journal of Veterinary Diagnostic Investigation 22(1), 61-66.

Sakai-Tagawa, Y., Ozawa, M., Yamada, S., Uchida, Y., Saito, T., Takahashi, K., Sugaya, N., Tashiro, M. and Kawaoka, Y. (2014). Detection sensitivity of influenza rapid diagnostic tests. Microbiology and Immunology, 58(10), 600-606.

Sandbulte, M. R., Gao, J., Straight, T. M. and Eichelberger, M. C. (2009). A miniaturized assay for influenza neuraminidase-inhibiting antibodies utilizing reverse genetics-derived antigens. Influenza and Other Respiratory Viruses 3(5), 233-240.

Shan, S., Ko, L. S., Collins, R. A., Wu, Z., Chen, J., Chan, K. Y., Xing, J., Lau, L. T. and Yu, A. C. (2003). Comparison of nucleic acid-based detection of avian influenza H5N1 with virus isolation. Biochemical and Biophysical Research Communications 302(2), 377-383.

Shi, P., Geng, S., Li, T.-t., Li, Y.-s., Feng, T. and Wu, H.-n. (2015). Methods to detect avian influenza virus for food safety surveillance. Journal of Integrative Agriculture 14(11), 2296-2308.

Spackman, E. and Suarez, D. L. (2008). Type A influenza virus detection and quantitation by real-time RT-PCR. Methods in Molecular Biology, 436, $19-26$.

Suarez, D. L., Das, A. and Ellis, E. (2007). Review of rapid molecular diagnostic tools for avian influenza virus. Avian Diseases 51(1 Suppl), 201-208.

Tahir, M. S., Mehmood, D., Sultan, A. U., Saeed, M. H., Khan, A. R., Ansari, F., Salman, M. M. and Majeed, K. A. (2016). A modified strategy of multiplex RT-PCR for simultaneous detection of $\mathrm{H} 5, \mathrm{H} 7$, and $\mathrm{H} 9$ subtypes of avian influenza virus based on common forward oligo. The Journal of Applied Poultry Research 25(3), 322-327.

Tavakoli, A., Rezaei, F., Fatemi Nasab, G. S., Adjaminezhad-Fard, F., Noroozbabaei, Z. and Mokhtari-Azad, T. (2017). The comparison of sensitivity and specificity of ELISA-based microneutralization test with hemagglutination inhibition test to evaluate neutralizing antibody against influenza virus (H1N1). Iran Journal of Public Health 46(12), 1690-1696.

Terregino, C. and Capua, I. (2009). Conventional diagnosis of avian influenza. In: Avian Influenza and Newcastle Disease: A Field and Laboratory Manual. Capua, I. and Alexander, D. J. (eds.). Springer Milan, Milano. pp 73-85.

Thi Nguyen, D., Shepard, S. S., Burke, D. F., Jones, J., Thor, S., Nguyen, L. V., Nguyen, T. D., Balish, A., Hoang, D. N., To, T. L., Iqbal, M., Wentworth, D. E., Spackman, E., van Doorn, H. R., Davis, C. T. and Bryant, J. E. (2018). Antigenic characterization of highly pathogenic avian influenza $A(H 5 N 1)$ viruses with chicken and ferret antisera reveals clade- 
dependent variation in hemagglutination inhibition profiles. Emerging Microbes \& Infections 7(1), 100.

Townsend, M. B., Dawson, E. D., Mehlmann, M., Smagala, J. A., Dankbar, D. M., Moore, C. L., Smith, C. B., Cox, N. J., Kuchta, R. D. and Rowlen, K. L. (2006). Experimental evaluation of the FluChip diagnostic microarray for influenza virus surveillance. Journal of clinical microbiology 44(8), 2863-2871.

Van Deusen, R., Hinshaw, V., A Senne, D. and Pellacani, D. (1983). Micro neuraminidase-inhibition assay for classification of influenza $A$ virus neuraminidases. Avian diseases 27(3),745-750.

Van Zyl, G. (2006). Laboratory Findings. In: Influenza Report 2006. Kamps, B. S., Hoffmann, C. and Preiser, P. (eds.). Flying Publisher. pp 150-159.

Vandalen, K. K., Shriner, S. A., Sullivan, H. J., Root, J. J. and Franklin, A. B. (2009). Monitoring exposure to avian influenza viruses in wild mammals. Mammal Review, 39(3), 167-177.

Vemula, S. V., Zhao, J., Liu, J., Wang, X., Biswas, S. and Hewlett, I. (2016). Current approaches for diagnosis of influenza virus infections in humans. Viruses, 8(4), 96.

Wang, C., Zhang, Y., Bing, G., Zhang, X., Wang, C., Wang, M., Sun, Y., Wu, S., Lin, X., Pu, J., Liu, J. and Sun, H. (2018). The use of pyrosequencing for detection of hemagglutinin mutations associated with increased pathogenicity of $\mathrm{H} 5 \mathrm{~N} 1$ avian influenza viruses in mammals. Journal of Veterinary Diagnostic Investigation 30(4), 619-622.

Wang, R. and Li, Y. (2013). Hydrogel based QCM aptasensor for detection of avian influenza virus. Biosensors and Bioelectronics 42, 148-155.

Wang, R. and Li, Y. (2016) Biosensors for rapid detection of avian influenza. In: Steps Forwards in Diagnosing and Controlling Influenza. Baddour, M. M. (eds.) InTech, Croatia. pp. 61-84.

Wang, R., Wang, L., Callaway, Z. T., Lu, H., Huang, T. J. and Li, Y. (2017). A nanowell-based QCM aptasensor for rapid and sensitive detection of avian influenza virus. Sensors and Actuators B: Chemical 240, 934-940.

WHO. (2005). Avian influenza: Assessing the pandemic threat. [Online] Available from: https://www.who.int/influenza/resources/documents/h5 n1_assessing_pandemic_threat/en/ [Accessed 18 Sept 2019].

WHO. (2010). Serological diagnosis of influenza by microneutralization assay. [Online] Available from: http://www.who.int/influenza/gisrs_laboratory/2010_12 06_serological_diagnosis_of_influenza_by_microneu tralization_assay.pdf [Accessed 18 Sept 2019].

WHO. (2011). WHO global influenza surveillance network: Manual for the laboratory diagnosis and virological surveillance of influenza. WHO Press, Switzerland.

WHO. (2017). Analysis of Recent Scientific Information on Avian Influenza A(H7N9) Virus. [Online] Available from:https://www.who.int/influenza/human_animal_int
erface/avian_influenza/riskassessment_AH7N9_2017 02/en/ [Accessed 18 Sept 2019 ].

Wibowo, M., Untari, T., Artanto, S., Putri, K., Amanu, S. and Asmara, W. (2017). Evaluation of rapid detection kit against avian influenza $A$ Virus and H5 Subtype for field sample. Indonesian Journal of Biotechnology 21(1), 48.

Wilson, G., Ye, Z., Xie, H., Vahl, S., Dawson, E. and Rowlen, K. (2017). Automated interpretation of influenza hemagglutination inhibition (HAl) assays: Is plate tilting necessary? PloS One 12(6), e0179939e0179939.

Woolcock, P. R. and Cardona, C. J. (2005). Commercial immunoassay kits for the detection of influenza virus type A: Evaluation of their use with poultry. Avian Diseases 49(4), 477-481.

Woolcock, P. R. (2008). Avian influenza virus isolation and propagation in chicken eggs. In: Avian Influenza Virus. Methods in Molecular Biology ${ }^{\mathrm{TM}}$, vol. 436. Spackman E. (eds). Humana Press. pp 35-46.

Yeo, S. J., Choi, K., Cuc, B. T., Hong, N. N., Bao, D. T., Ngoc, N. M., Le, M. Q., Hang Nle, K., Thach, N. C., Mallik, S. K., Kim, H. S., Chong, C. K., Choi, H. S., Sung, H. W., Yu, K. and Park, H. (2016a). Smartphone-based fluorescent diagnostic system for highly pathogenic H5N1 viruses. Theranostics 6(2), 231-242.

Yeo, S. J., Cuc, B. T., Sung, H. W. and Park, H. (2016b). Evaluation of a smartphone-based rapid fluorescent diagnostic system for H9N2 virus in specific-pathogen-free chickens. Archives of Virology 161(8), 2249-2256.

Yu, H., Cowling, B. J., Feng, L., Lau, E. H., Liao, Q., Tsang, T. K., Peng, Z., Wu, P., Liu, F., Fang, V. J., Zhang, H., Li, M., Zeng, L., Xu, Z., Li, Z., Luo, H., Li, Q., Feng, Z., Cao, B., Yang, W., Wu, J. T., Wang, Y. and Leung, G. M. (2013). Human infection with avian influenza A H7N9 virus: An assessment of clinical severity. Lancet 382(9887), 138-145.

Zhao, J., Ragupathy, V., Liu, J., Wang, X., Vemula, S. V., El Mubarak, H. S., Ye, Z., Landry, M. L. and Hewlett, I. (2015). Nanomicroarray and multiplex Next-Generation sequencing for simultaneous identification and characterization of influenza viruses. Emerging of Infectious Diseases 21(3), 400-408.

Zhao, J., Tang, S., Storhoff, J., Marla, S., Bao, Y. P., Wang, X., Wong, E. Y., Ragupathy, V., Ye, Z. and Hewlett, I. K. (2010). Multiplexed, rapid detection of H5N1 using a PCR-free nanoparticle-based genomic microarray assay. BMC Biotechnology 10(1), 74. 